\title{
Síndrome de Burnout e Síndrome do Impostor: um estudo correlacional
}

\author{
Burnout's Syndrome and Impostor Syndrome: a correlational study \\ Síndrome de Burnout y Síndrome del Impostor: un estudio correlacional
}

Recebido: 26/02/2021 | Revisado: 07/03/2021 | Aceito: 10/03/2021 | Publicado: 18/03/2021

Aldení Ramos de Oliveira
ORCID: https://orcid.org/0000-0001-5772-610X
Centro Universitário de Patos, Brasil
E-mail: aldeniramos59@ gmail.com
Thereza Christina Garcia Bezerra
ORCID: https://orcid.org/0000-0002-9014-5697
Centro Universitário de Patos, Brasil
E-mail: tchristinapsico@ gmail.com
Tessya Hyanna Almeida Oliveira
ORCID: https://orcid.org/0000-0003-1562-1478
Centro Universitário de Patos, Brasil
E-mail: tessyahyanna @ outlook.com
Alessandro Teixeira Rezende
ORCID: https://orcid.org/0000-0002-5381-2155
Universidade Federal da Paraíba, Brasil
E-mail: alessandro.teixeira.rezende @ gmail.com
Camilla Vieira de Figueiredo
ORCID: https://orcid.org/0000-0001-9780-9831
Universidade Federal da Paraíba, Brasil
E-mail: camillafigueir@gmail.com
Tarciana Sampaio Costa
Centro Universitário de Patos, Brasil
E-mail: tarcianacosta @ fiponline.edu.br

\section{Resumo}

Objetivo: O presente estudo objetivou demonstrar a relação entre a Síndrome do Impostor e a Síndrome de Burnout no contexto de estudo e atuação profissional na área de Enfermagem. Metodologia: Realizou-se um estudo descritivo, de caráter quantitativo e correlacional, do qual participaram 106 pessoas, sendo estas acadêmicos de Enfermagem $(60,4 \%)$ e profissionais da Enfermagem (39,6\%). Estes responderam a Escala de Burnout de Maslach e a Escala Clance do Fenômeno Impostor. Resultados: Constatou-se que os participantes apresentaram escores médios superiores para a Síndrome de Burnout em relação à Síndrome do Impostor. Além disso, observaram-se correlações positivas e significativas entre o fator geral da Síndrome do Impostor e os fatores despersonalização $(r=0,57 ; p<$ $0,01)$ e exaustão emocional $(r=0,44 ; p<0,01)$, característicos da Síndrome de Burnout, bem como com o fator geral de Burnout $(r=0,51 ; p<0,01)$. Conclusão: As síndromes investigadas neste estudo têm implicações significativas para a saúde física e mental dos indivíduos e para o seu bem estar em geral. Confia-se que os resultados obtidos podem direcionar novas investigações sobre a temática e, posteriormente, fundamentar o desenvolvimento de estratégias de intervenção em relação às Síndromes de Burnout e do Impostor no contexto dos profissionais que atuam na saúde.

Palavras-chave: Burnout; Síndrome do impostor; Enfermagem.

\begin{abstract}
Objective: The present study aimed to demonstrate the relationship between Impostor Syndrome and Burnout Syndrome in the context of study and professional performance in the area of Nursing. Methodology: For this purpose, a descriptive study was carried out, with a quantitative and correlational character, in which 106 people participated, being these Nursing students (60.4\%) and Nursing professionals (39.6\%). These responded to the Maslach Burnout Inventory and Clance Imposter Phenomenon Scale. Results: As a result, it was found that the participants had higher mean scores for the Burnout Syndrome compared to the Impostor Syndrome. In addition, there were positive and significant correlations between the general factor of the Impostor Syndrome and the factors depersonalization $(\mathrm{r}=.57 ; \mathrm{p}<.01)$ and emotional exhaustion $(\mathrm{r}=.44 ; \mathrm{p}<.01)$, characteristic of the Burnout Syndrome, as well as with the general Burnout factor $(r=.51 ; p<.01)$. Conclusion: It is argued that the syndromes investigated in this study have significant implications for the physical and mental health of individuals and their wellbeing in general. It is hoped that the results obtained will direct new investigations on the theme and, later, support the
\end{abstract}


development of intervention strategies to Burnout and Impostor Syndromes in the context of professionals working in health.

Keywords: Burnout; Impostor syndrome; Nursing.

\section{Resumen}

Objetivo: El presente estudio tuvo como objetivo demostrar la relación entre el Síndrome del Impostor y el Síndrome de Burnout en el contexto de estudio y desempeño profesional en el campo de la Enfermería. Metodología: Para ello se realizó un estudio descriptivo, de carácter cuantitativo y correlacional, en el que participaron 106 personas, siendo estos estudiantes de Enfermería (60,4\%) y Profesionales de Enfermería (39,6\%). Estos respondieron al Inventario de Burnout de Maslach y la Escala de Fenómeno de Clance Imposter. Resultado: Como resultado, se encontró que los participantes tenían puntuaciones medias más altas para el Síndrome de Burnout en comparación con el Síndrome del Impostor. Además, se encontraron correlaciones positivas y significativas entre el factor general del Síndrome del Impostor y los factores despersonalización $(r=0,57 ; p<0,01)$ y agotamiento emocional $(r=0,44 ; p<0,01)$, característico del Burnout. Síndrome, así como con el factor Burnout general $(r=0,51 ; p<0,01)$. Conclusión: Se argumenta que los síndromes investigados en este estudio tienen implicaciones significativas para la salud física y mental de las personas y su bienestar en general. Se espera que los resultados obtenidos orienten nuevas investigaciones sobre el tema y, posteriormente, apoyen el desarrollo de estrategias de intervención a los Síndromes de Burnout e Impostor en el contexto de los profesionales que trabajan en salud.

Palabras clave: Burnout; Sindrome del impostor; Enfermería.

\section{Introdução}

O adoecimento psíquico relacionado ao trabalho é um fenômeno crescente na atualidade. Modos de organização inadequados e alta demanda de atividades e atribuições acarretam significativos riscos para a saúde e para o bem estar biopsicossocial dos indivíduos, aumentando os seus níveis de ansiedade, depressão e estresse. Em contexto laboral, esses aspectos interferem diretamente na saúde física e psicológica dos trabalhadores, bem como nas relações e no envolvimento dos mesmos com o seu trabalho (Hazlett-Stevens, 2012).

Nesse cenário, um fenômeno que merece destaque é o estresse. Atualmente, considerando as crescentes pressões a que os trabalhadores estão submetidos, bem como as sucessivas mudanças no contexto do trabalho, os profissionais enfrentam cargas de trabalho exacerbadas, acrescidas de grandes cobranças por produtividade, o que gera sobrecarga e tensão e, consequentemente, constitui-se como terreno fértil para o desenvolvimento do estresse. Quando expostos ao estresse em alta intensidade, os trabalhadores podem sentir grandes impactos na sua saúde, como desânimo, ansiedade e exaustão emocional, fatores que caracterizam o estresse ocupacional (Silva, Ferreira, Albuquerque, Rodrigues, \& Medeiros, 2016).

O estresse ocupacional se evidencia a curto e longo prazo no trabalhador, que passa a se sentir pressionado e ameaçado por ações estressoras no ambiente laboral. Essas ações geram instabilidade no trabalho, desmotivação na prestação dos serviços, dificuldades nos relacionamentos interpessoais, sensação de desconfiança, assédio moral, baixa autoestima e queda de rendimento, acarretando significativos prejuízos à saúde do trabalhor, bem como rotatividade e comprometimento na parte organizacional (Cooper, 2007; Paiva, Gomes, \& Helal, 2015). Além disso, há evidências de que o estresse desencadeia síndromes e psicopatologias. Nessa lógica, quando os seus sinais e sintomas se tornam crônicos, causando a completa exaustão profissional, o estresse passa a ser caracterizado como Síndrome de Burnout (SB) (Santana \& Tonon, 2020).

A SB, do inglês "burn", que significa queima, e "out" que significa fora, indicando a ideia de "queimar por completo", é definida como algo que deixou de funcionar por completo devido a falta de energia. A SB, qualificada como uma síndrome ocupacional, decorre de situações vivenciadas no ambiente de trabalho ligadas principalmente à pressão e ao excesso de obrigações contínuas que ocasionam um total esgotamento do trabalhador (Monteiro, 2020). Atualmente, a SB apresenta uma incidência expressiva no cenário mundial, especialmente no Brasil.

De acordo com a International Stress Management Association (ISMA, 2017), as estatísticas afirmam que os níveis sintomatológicos de Burnout, em escala mundial, no ranking de oito países, são mais acentuados no Japão e, em segundo lugar, no Brasil, onde mais de $70 \%$ dos trabalhadores desenvolvem algum tipo de acometimento em consequência do estresse 
ocupacional (Silveira et al., 2016). As evidências apontam ainda que, de maneira geral, a SB acomete 32\% dos trabalhadores. No entanto, dados mais específicos indicam que destes trabalhadores, os profissionais da área da Enfermagem apresentam maiores predisposições aos sintomas do Burnout e que o princípio da síndrome pode ocorrer ainda na fase acadêmicoestudantil do curso de Enfermagem (Jantsch, Costa, \& Pissaia, 2018).

Há certo consenso por caracterizar a SB como a junção dos sintomas de exaustão emocional, despersonalização e diminuição da realização profissional, os quais formam uma tríade representativa do construto Burnout amplamente aceita pelos pesquisadores da saúde (Benevides-Pereira, 2002). Conceptualmente, a SB é complexa, multidimensional e pode estar ligada a situações específicas que consolidam a influência direta entre o trabalho e o adoecimento psicossocial. Para além de fatores como o estresse, a ansiedade e a depressão, já mencionados, a SB pode favorecer o desenvolvimento de comportamentos de ideação suicida. Sintomatologias de natureza psicossocial, psicossomática, comportamental e defensiva podem desencadear a SB, a qual pode ainda se relacionar com características de outras síndromes, a exemplo da Síndrome do Impostor (SI).

A SI foi descrita inicialmente por Clance e Imes (1978), que observaram um grupo de mulheres bem-sucedidas tentando explicar as crenças e sentimentos de que o próprio sucesso era fruto de sorte e da visão equivocada que as outras pessoas tinham a respeito de suas competências. Assim, o sujeito que sofre da SI se considera uma fraude, especialmente no campo profissional, e acredita não merecer o sucesso e status alcançados, além de conviver com o medo de ser percebido pelos outros dessa maneira. A SI tende a surgir no contexto acadêmico, podendo causar grandes prejuízos à carreira profissional (Parkman, 2016).

Alguns comportamentos específicos relacionados à SI e que dificultam a sua melhora têm sido investigados. O primeiro comportamento é aquele em que a pessoa demonstra interesse, trabalho árduo e esforços que ultrapassam o esperado na busca por esconder o seu sentimento de falta de capacidade. Outro tipo de comportamento remete a um falso intelecto, de maneira que a pessoa usa um viés tendencioso para apresentar suas ideias, passando a crer que se tivesse sugerido a sua ideia de fato esta não seria aceita (Bezerra et al., 2020). Uma terceira modalidade de comportamento relacionada à SI é a de que a pessoa usa de artimanhas sensuais ou bajulação para chamar atenção de seus superiores, pois acredita que só conseguirá o sucesso desejado utilizando tais artifícios, deslegitimando, assim, as suas competências. Finalmente, pesquisadores da SI levantaram que a síndrome engloba ainda comportamentos nos quais a pessoa finge não ser competente e evita ser bemsucedida porque acredita que seria hostilizada no seu convívio social (Parkman, 2016).

A SB e a SI são consideradas problemas graves de saúde, pois tratam-se de acometimentos que geram consequências para a saúde física e psicológica dos trabalhadores em geral e dos profissionais de saúde em particular. Estes últimos enfrentam cotidianamente grandes desafios relacionados ao trabalho nos contextos de saúde, vivenciando um contato direto e diário com pacientes em processos de saúde, de doença, de perdas e de morte, efeitos que influenciam sobremaneira as diversas esferas da vida do profissional de saúde, em especial daqueles da área da Enfermagem (Andrade \& Siqueira Junior, 2014).

A literatura tem demonstrado que os grupos mais vulneráveis a experienciar sintomas de ambas as síndromes são professores (Abacar, Aliante, \& António, 2020; Diehl \& Carlotto, 2020; Silva \& Oliveira, 2019), enfermeiros (Ramos, Farias, Costa, \& Fonseca, 2019; Sé et al., 2020), psicólogos (Rodriguez, Carlotto, \& Câmara, 2017), assistentes sociais (Perniciotti, Serrano Júnior, Guarita, Morales, \& Romano, 2020), policiais (Lima, Lima, Oliveira, Ferreira, \& Pacheco Neto, 2018), médicos (Moreira, Souza, \& Yamaguchi, 2018), fisioterapeutas (Silva et al., 2018) e acadêmicos (Carlotto \& Câmara, 2020; Lopes \& Nihei, 2020). Observa-se que o desenvolvimento de sintomas, tanto da SB como da SI, pode decorrer de contextos laborais, acadêmicos, mas também de recorrentes e adoecedoras situações do meio social em geral. O predomínio e a extensão de tais sintomas dependem da resposta de cada indivíduo ao seu meio, bem como das dúvidas, dos níveis de estresse e ansiedade e das estratégias de enfrentamento utilizadas (Batista, Carlotto, Coutinho, \& Augusto, 2010). 
Apesar da relevância de se investigar a relação entre estas síndromes, são escassos os estudos com essa finalidade na literatura nacional atual. Diante desse quadro, o presente estudo tem como objetivo conhecer como se relacionam a SI e a SB entre acadêmicos e profissionais de Enfermagem. Considera-se que essa proposta tem grande relevância para a literatura da área, podendo direcionar novas investigações sobre a temática e, posteriormente, servir de insumo para o desenvolvimento de estratégias de intervenção voltadas para a promoção e prevenção da saúde mental dos trabalhadores, bem como para o aumento da qualidade de vida de profissionais de saúde acometidos por tais síndromes.

\section{Metodologia}

\section{Delineamento e participantes}

Trata-se de um estudo descritivo, de caráter quantitativo e correlacional (ex post facto), do qual participaram 106 pessoas, dentre as quais estudantes de Enfermagem $(60,4 \%)$ e profissionais de Enfermagem $(39,6 \%)$, com média de idade de 30 anos $(D P=6,19)$. A maioria autodeclarou-se do sexo feminino $(78,1 \%)$, solteira $(55,7 \%)$ e de classe social média $(42,5 \%)$. É importante destacar que se tratou de amostra de conveniência, participando do estudo aqueles que, convidados, o fizeram voluntariamente.

\section{Instrumentos}

Além de questões sociodemográficas (e.g., sexo, idade, classe socioeconômica), os participantes responderam os seguintes instrumentos:

Escala de Burnout de Maslach. Elaborada por Maslach e Jackson (1978) e originalmente intitulada Maslach Burnout Inventory, trata-se de um instrumento frequentemente utilizado para mensurar a SB. Tal medida foi adaptada e validada para o contexto brasileiro por Tamayo (1997) e conta com 22 itens que representam três dimensões específicas da SB: exaustão emocional, despersonalização e, inversamente, realização profissional. As respostas ao instrumento são fornecidas em escala Likert de 7 pontos, variando de 0 (Nunca) a 6 (Todos os dias). Assim, pontuações mais altas em exaustão emocional e despersonalização associadas a pontuações baixas em realização profissional indicam que o indivíduo experiencia com maior frequência características da SB.

Escala Clance do Fenômeno Impostor. Foi desenvolvida originalmente na língua inglesa por Clance (1985), sendo adaptada para o contexto brasileiro por Bezerra, Barbosa, Vione, Athayde e Gouveia (2020). A escala original apresentou uma estrutura trifatorial, incluindo os fatores de "Medo de ser incapaz de repetir o sucesso", "Medo de ser avaliado" e "Medo de ser menos capaz que os outros" (Clance, 1985). No entanto, a versão brasileira desta medida, utilizada no presente estudo, apresentou uma estrutura unifatorial. O instrumento é composto por 20 itens e as respostas são fornecidas em escala Likert de 5 pontos, variando de 1 (Não me descreve) a 5 (Me descreve totalmente). Desse modo, pontuações mais altas indicam que o indivíduo experiencia mais características da SI.

\section{Análise de dados}

Os dados foram organizados e analisados por meio do SPSS, em sua versão 21. Foram realizadas estatísticas descritivas (e.g., média, frequência, desvio padrão) e correlações $r$ de Pearson para conhecer as relações entre as variáveis.

\section{Procedimentos e aspectos éticos}

Inicialmente, o projeto que compreende essa pesquisa foi submetido ao Comitê de Ética de uma instituição brasileira de ensino superior. Após a sua aprovação (parecer no 4.306.920/CAAE 37285220.0000.5181), iniciou-se a coleta dos dados, que ocorreu durante os meses de outubro e dezembro de 2020. A coleta procedeu de forma online por meio de um questionário 
desenvolvido no Google Docs. Nesse questionário de pesquisa estavam dispostos os objetivos, procedimentos, bem como explicações sobre o caráter sigiloso e voluntário da participação. Os acadêmicos e profissionais de Enfermagem foram convidados a participar da pesquisa por meio de anúncios divulgados nas redes sociais Whatsapp, Facebook e Instagram. Para firmar a sua participação, os respondentes tinham de ler e concordar com um Termo de Consentimento Livre e Esclarecido (TCLE), apresentado assim que os participantes clicavam no link da pesquisa. O TCLE indicava que eles autorizavam o uso dos dados pelos pesquisadores para finalidades científicas, como também que poderiam desistir da participação na pesquisa em qualquer momento da mesma, sem quaisquer prejuízos. Dessa forma, buscou-se atender todos os critérios éticos dispostos na Resolução no 466/2012 do Conselho Nacional de Saúde. O tempo médio para que concluíssem o questionário foi de 20 minutos.

\section{Resultados}

Inicialmente, calculou-se as pontuações totais dos respondentes nos fatores gerais de SB e de SI. Os dados foram analisados conjuntamente para acadêmicos e profissionais. Quanto ao escore médio no fator geral de SI, os participantes obtiveram uma média de 2,53 ( $D P=0,94)$. Na SB, por sua vez, considerando o comput total dos três fatores que a compõem, os participantes apresentaram uma média geral de 2,70 $(D P=0,79)$. Em seguida, computou-se separadamente os três componentes específicos da SB a fim de conhecer as pontuações médias dos participantes em cada um deles. Os resultados indicaram que os participantes pontuaram mais alto no fator de SB correspondente à exaustão emocional $(M=3,44 ; D P=$ $1,21)$, seguido pelo fator realização profissional $(M=2,37 ; D P=1,50)$ e, por fim, pelo fator despersonalização $(M=2,29$; $D P=1,35)$. De maneira geral, estes resultados indicam que as pontuações médias dos participantes foram ligeiramente mais elevadas para a SB do que para a SI.

Em seguida, buscou-se conhecer a relação entre o fator geral da SI com os fatores específicos da SB. Os resultados indicaram correlações positivas e significativas entre a SI e os fatores da SB despersonalização $(r=0,57 ; p<0,001) \mathrm{e}$ exaustão emocional ( $r=0,44 ; p<0,001)$, bem como com o fator geral da SB $(r=0,51 ; p<0,001)$. No entanto, a SI não logrou relação significativa com o fator realização profissional da SB $(p>0,05)$. Estes resultados são apresentados na Tabela 1.

Tabela 1. Relação entre Síndrome do Impostor e Síndrome de Burnout.

\begin{tabular}{lcccc}
\hline \multicolumn{1}{c}{ Fator } & SI & EE & RP & D \\
\hline Exaustão emocional (EE) & $0,44^{* *}$ & & & \\
Realização profissional (RP) & $-0,05$ & $-0,35^{* *}$ & & \\
Despersonalização (D) & $0,57^{* *}$ & $0,62^{* *}$ & $-0,16$ & \\
Fator geral de Burnout & $0,51^{* *}$ & $0,65^{* *}$ & $0,36^{* *}$ & $0,79 * *$ \\
\hline
\end{tabular}

Nota: SI = Fator geral de Síndrome do Impostor; $* * p<0,01$. Fonte: Autoria própria.

\section{Discussão}

O presente estudo teve como principal objetivo conhecer como se relacionam a SI e a SB entre estudantes e profissionais da área da Enfermagem. Especificamente, os resultados permitiram identificar os níveis de SB e de SI dos participantes e, de maneira geral, indicaram que a presente amostra alcançou escores ligeiramente mais elevados para SB. Os resultados obtidos através da Escala de Burnout de Maslach apontaram pontuações mais elevadas no fator específico nomeado como exaustão emocional. Esse fator remete à sensação que o indivíduo tem de estar ultrapassando seus limites físicos e psicológicos. Essa sensação está relacionada à sobrecarga de trabalho e ao esgotamento físico e mental, o que concorre para 
que o indivíduo não se sinta com energia suficiente para desempenhar suas atividades laborais (Pêgo \& Pêgo, 2016). Escores mais elevados nesta dimensão do Burnout foram esperados para a presente amostra em virtude especialmente de a pesquisa ter sido realizada no ano de 2020, período marcado pela pandemia da COVID-19 e pela atuação dos profissionais de Enfermagem na linha de frente do combate ao vírus.

Na sequência, o fator de SB nomeado realização profissional foi o segundo mais endossado pelos acadêmicos e profissionais de Enfermagem. A realização profissional pode atuar como um fator protetivo do desenvolvimento da SB, estando relacionada às recompensas advindas da formação, como possibilidade de crescimento na carreira, conquista do respeito da chefia e dos colegas de trabalho, suporte das pessoas em momentos difíceis e recebimento de tratamento justo (Bezerra et al., 2020). Finalmente, o fator despersonalização foi o menos endossado pela amostra desse estudo, sendo caracterizado por comportamentos negativos do profissional para com seus colegas de trabalho e por reações de insensibilidade e indiferença adquiridas como respostas à exaustão profissional. Resultados recentemente publicados por Valério et al. (2020) contrastam com os nossos, de maneira que a amostra considerada por estes autores endossou em maior medida o fator de realização profissional em comparação aos demais fatores característicos da SB. Especificamente, no estudo desses autores a realização profissional caracterizou 75,6\% da amostra, despersonalização 60,3\% e exaustão emocional 53,8\%.

O contexto de enfrentamento da pandemia da COVID-19 por parte dos profissionais de Enfermagem brasileiros pode potencialmente explicar essa diferença. Os altos índices de desgaste físico e psicológico desses trabalhadores, a ausência de condições salubres e ambientes físicos de trabalho viáveis, bem como a expressiva desvalorização financeira e social da profissão são alguns dos fatores que os enfermeiros brasileiros têm de enfrentar diariamente. Esses aspectos são comuns em situações de normalidade (Pereira, Torres, Pereira, Antunes, \& Costa, 2020). Nos dias atuais, porém, os profissionais da Enfermagem vem se deparando com desafios ainda maiores impostos pela pandemia da COVID-19. Estes desafios remetem principalmente ao alto risco de infecção pelo vírus, de adoecimento e até morte, à chance de infectar outros indivíduos, exposição a mortes em ampliadas proporções, frustração por não conseguir salvar vidas e pelo limite de recursos na saúde pública brasileira, distanciamento de amigos e familiares, dentre outros aspectos que podem explicar o aumento de sentimentos de angústia e exaustão física e emocional em detrimento da diminuição de sentimentos de realização profissional (Lancet, 2020).

No que se refere à SI, os resultados indicaram que o escore médio dos participantes situou-se exatamente no ponto médio da escala ( $M=2,53$, sendo a escala de resposta de 1 a 5). Percepção inadequada e negativa acerca do próprio sucesso, crenças distorcidas de não merecimento desse sucesso, além da visão de que o mesmo é oriundo de sorte ou fatores do acaso, faz com que os sujeitos que sofrem com a SI acreditem que são uma fraude (Parkman, 2016). Sobre as correlações evidenciadas no presente estudo, observou-se uma correlação positiva entre a SI e o fator exaustão emocional, característico da SB. Com base no estudo de Clance e Imes (1978), o indivíduo que pontua alto em características da SI possui uma visão equivocada acerca de si e de suas conquistas, o que faz com que experiencie sintomas semelhantes aos da exaustão emocional, como ansiedade, estresse e sofrimento psicológico.

No que diz respeito especificamente à relação entre SI e o fator despersonalização, os resultados também apontaram uma relação positiva. Essa relação pode ser explicada pelo fato de que os indivíduos que sofrem com a SI podem, perante os colegas, serem considerados falsos ou cínicos, uma vez que podem criar ou endossar determinadas posturas no ambiente de trabalho com o intuito de que os colegas não percebam os seus sentimentos de incapacidade e incompetência (Clance \& Imes, 1978). Em resumo, os resultados do presente estudo são claros sobre a relação entre as síndromes em questão, demonstrando que quanto quanto mais o indivíduo experiencia sintomas da SI, mais se sente esgotado emocionalmente $(r=0,44 ; p<0,01) \mathrm{e}$ indiferente ou despersonalizado em relação ao ambiente laboral $(r=0,57 ; p<0,01)$.

Não obstante, no que tange ao fator de realização profissional, observou-se a ausência de correlação estatisticamente 
significativa entre ele e o fator geral de SI. Embora esperasse-se uma correlação negativa entre essas variáveis, de maneira que quanto mais o indivíduo apresenta características da SI, menos sente-se realizado profissionalmente, essa relação não foi observada para a presente amostra. A realização profissional está relacionada à percepção de bom desempenho no trabalho e de que este desempenho é recompensado por uma remuneração justa e por oportunidades de crescimento profissional (Oliveira et al., 2019). Dessa forma, estimou-se que a realização profissional poderia estar inversamente associada a características da SI, já que o indivíduo com sentimentos de impostor costuma ter uma percepção equivocada acerca de seu desempenho, acreditando que suas conquistas, habilidades e sucesso podem ser atribuídas à sorte, mesmo que as evidências contradigam objetivamente essa percepção (Simon \& Choi, 2018). Estes resultados sugerem a necessidade de investigar de forma mais aprofundada, inclusive considerando diferentes amostras, a relação entre realização profissional e SI.

\section{Considerações Finais}

O presente estudo objetivou conhecer a relação entre a SI e os componentes característicos da SB para uma amostra de profissionais e estudantes de Enfermagem. Confia-se que tal objetivo tenha sido alcançado, uma vez que os resultados indicaram relações fortes, positivas e significativas entre a SI e os componentes do Burnout. Não obstante, é certo que estes resultados não estão isentos de limitações, as quais correspondem em maior medida à amostra considerada, composta majoritariamente por acadêmicos. Nesse sentido, sugere-se a ampliação desta amostra em estudos futuros, tornando-a equitativa, além de estabelecer novos objetivos de pesquisa, como buscar conhecer a relação entre a SB e a SI com outros construtos psicológicos, a exemplo dos traços personalidade e os valores humanos. Ainda, sugere-se o desenvolvimento de modelos explicativos envolvendo a SB e a SI que possam indicar a direção da relação e o poder preditivo dessas variáveis. Por fim, estima-se que o presente estudo, além de fornecer dados empíricos acerca de uma temática ainda escassa à nível nacional, pode fundamentar o desenvolvimento de outras pesquisas, bem como a estruturação de estratégias de intervenção que visem a redução dos níveis de SB e de SI de acadêmicos e profissionais de Enfermagem, como também de outros profissionais que atuam nos contextos de saúde.

\section{Referências}

Abacar, M., Aliante, G., \& António, F. (2020). Burnout em professores do ensino secundário. Research, Society and Development, 9(7), e545973776e545973776.

Andrade, M. C. M, \& Siqueira Junior, A. C. (2014). Estresse ocupacional no serviço de atendimento móvel de urgência. Rev Min. Enferma, 18(2), 376-383.

Batista, J. B. V., Carlotto, M. S., Coutinho, A. S., \& Augusto, L. G. D. S. (2010). Prevalência da Síndrome de Burnout e fatores sociodemográficos e laborais em professores de escolas municipais da cidade de João Pessoa/PB. Revista Brasileira de Epidemiologia, 13(3), 502-512.

Benevides-Pereira, A. M. T. (2002). Burnout: O Processo de Adoecer Pelo Trabalho. In A. M. T. Benevides-Pereira, Burnout: quando o trabalho ameaça o bem-estar do trabalhador (pp. 21-92). São Paulo: Casa do Psicólogo.

Bezerra, T. C. G., Barbosa, L. H., G. M., Vione, K. C., Athayde, R. A. A., \& Gouveia, V. V. (no prelo). Escala Clance do Fenômeno Impostor: Adaptação brasileira. Psico-USF.

Carlotto, M. S., \& Câmara, S. G. (2020). Escala de Avaliação da Síndrome de Burnout em Estudantes Universitários (ESB-eu): construção e evidências de validade. Research, Society and Development, 9(7), e171974013-e171974013.

Clance, P. R., \& Imes, S. A. (1978). The imposter phenomenon in high achieving women: Dynamics and therapeutic intervention. Psychotherapy: Theory, Research \& Practice, 15(3), 241-247.

Cooper, C. L. (2007). A natureza mutante do trabalho: o novo contrato psicológico e os estressores associados. In A. M. Rossi, P. L. Perrewé, S. L. Sauter (Orgs.), Qualidade de vida no trabalho: perspectivas atuais da saúde ocupacional (pp. 3-8). Atlas.

Diehl, L., \& Carlotto, M. S. (2020). Síndrome de Burnout em professores: Diferenças entre níveis de ensino. Research, Society and Development, 9(5), e62952623-e62952623.

Hazlett-Stevens, H. (2012). Mindfulness-based stress reduction for comorbid anxiety and depression: case report and clinical considerations. The Journal of Nervous and Mental Disease, 200(11), 999-1003. 
International Stress Management Association (ISMA) (2017). Burnout: mais próximo do setor da saúde do que se imagina. http://www.ismabrasil.com.br/img/estresse105.pdf

Jantsch, N., Costa, A. E. K., \& Pissaia, L. F. (2018). Síndrome de Burnout: uma revisão integrativa. Research, Society and Development, 7(1), e1371191e1371191.

Lancet, T. (2020). COVID-19: protecting health-care workers [Editorial]. Elsevier Public Health Emergency Collection, $395(10228), 922$.

Lima, F. R. B., Lima, D. L. F., Oliveira, A. A. R., Ferreira, E. O., \& Pacheco Neto, P. (2018). Identificação preliminar da síndrome de burnout em policiais militares. Motricidade, 14(1), 150-156.

Lopes, A. R., \& Nihei, O. K. (2020). Burnout em estudantes de Enfermagem: preditores e associação com empatia e autoeficácia. Revista Brasileira de Enfermagem, 73(1), e20180280.

Monteiro, L. (2020). OMS classifica a Síndrome de Burnout como doença. Recuperado de https://super.abril.com.br/saude/oms-classifica-a-sindrome-deburnout-como-doenca/

Moreira, H. D. A., Souza, K. N. D., \& Yamaguchi, M. U. (2018). Síndrome de Burnout em médicos: uma revisão sistemática. Revista Brasileira de Saúde Ocupacional, 43(3), e3.

Oliveira, A. P. S. D., Oliveira, A. L. D. S., Prado, R. M. D., Vasconcelos, A. M. V., Silva, J. C. V. D., \& Oliveira, J. C. D. (2019). O esgotamento físico dos enfermeiros no setor de urgência e emergência: revisão integrativa. Nursing (Säo Paulo), 22(251), 2839-2843.

Paiva, K. C. M. D., Gomes, M. Â. D. N., \& Helal, D. H. (2015). Estresse ocupacional e síndrome de burnout: proposição de um modelo integrativo e perspectivas de pesquisa junto a docentes do ensino superior. Gestão \& Planejamento-G\&P, 16(3), 285-309.

Parkman, A. (2016). The imposter phenomenon in higher education: Incidence and impact. Journal of Higher Education Theory and Practice, 16(1), 51-61.

Pêgo, F. P. L., \& Pêgo, D. R. (2016). Síndrome de Burnout. Rev Bras Med Trab, 14(2), 171-176.

Pereira, M. D., Torres, E. C., Pereira, M. D., Antunes, P. F. S., \& Costa, C. F. T. (2020). Sofrimento emocional dos Enfermeiros no contexto hospitalar frente à pandemia de COVID-19. Research, Society and Development, 9(8), e67985121-e67985121.

Perniciotti, P., Serrano Júnior, C. V., Guarita, R. V., Morales, R. J., \& Romano, B. W. (2020). Síndrome de Burnout nos profi ssionais de saúde: atualização sobre definições, fatores de risco e estratégias de prevenção. Revista da Sociedade Brasileira de Psicologia Hospitalar, $23(1), 35-52$.

Ramos, C. E. B., Farias, J. A., Costa, M. B. D. S., \& Fonseca, L. D. C. T. D. (2019). Impactos da Síndrome de Burnout na qualidade de vida dos profissionais de enfermagem da atenção básica à saúde. Rev. bras. ciênc. saúde, 23(3), 285-296.

Rodriguez, S. Y. S., Carlotto, M. S., \& Câmara, S. G. (2017). Impacto da regulação de emoções no trabalho sobre as dimensões de Burnout em psicólogos: O papel moderador da autoeficácia. Análise psicológica, 35(2), 191-201.

Santana, R. E., \& Tonon, T. C. A. (2020). Estresse ocupacional: desequilíbrio no exercício profissional de Enfermagem. Research, Society and Development, 9(8), e222985674-e222985674.

Sé, A. C. S., Machado, W. C. A., Passos, J. P., Gonçalves, R. C. S., Cruz, V. V., Bittencourt, L. P., \& Figueiredo, N. M. A. (2020). Prevalência da síndrome de burnout em enfermeiros do atendimento pré-hospitalar. Research, Society and Development, 9(7), e940975265-e940975265.

Silva, N. C. D., Ferreira, J. V. B., Albuquerque, T. C., Rodrigues, M. R., \& Medeiros, M. F. (2016). Transtornos à saúde mental relacionados à intensa rotina de trabalho do enfermeiro: uma revisão bibliográfica. Revista Eletrônica Estácio Saúde, 5(2), 107-122.

Silva, R. A. D. D., Araújo, B., Morais, C. C. A., Campos, S. L., Andrade, A. D. D., \& Brandão, D. C. (2018). Síndrome de Burnout: realidade dos fisioterapeutas intensivistas? Fisioterapia e Pesquisa, 25(4), 388-394.

Silva, S. M. F., \& Oliveira, Á. D. F. (2019). Burnout em professores universitários do ensino particular. Psicologia Escolar e Educacional, 23 , e187785.

Silveira, A. D., Colleta, T. C. D., Ono, H. R. B., Woitas, L. R., Soares, S. H., Andrade, V. L. A., \& Araújo, L. D. (2016). Síndrome de Burnout: consequências e implicações de uma realidade cada vez mais prevalente na vida dos profissionais de saúde. Rev Bras Med Trab, 14(3), 275-84.

Simon, M., \& Choi, Y. J. (2018). Using factor analysis to validate the Clance Impostor Phenomenon Scale in sample of science, technology, engineering and mathematics doctoral students. Personality and Individual Differences, 121, 173-175.

Tamayo, R. M. (1997). Relação entre a Síndrome de Burnout e os valores organizacionais no pessoal de Enfermagem de dois hospitais públicos. Dissertação de Mestrado não publicada. Instituto de Psicologia, Universidade de Brasília, Brasília, DF.

Valério, R. L., Oliveira, E. B., Kestenberg, C. C. F., Paula, V. G., Dias, L. B. S., \& Oliveira, T. S. (2020). Exaustão emocional em enfermeiros residentes de unidades especializadas em hospital universitário. Research, Society and Development, 9(2), e198922240-e198922240. 